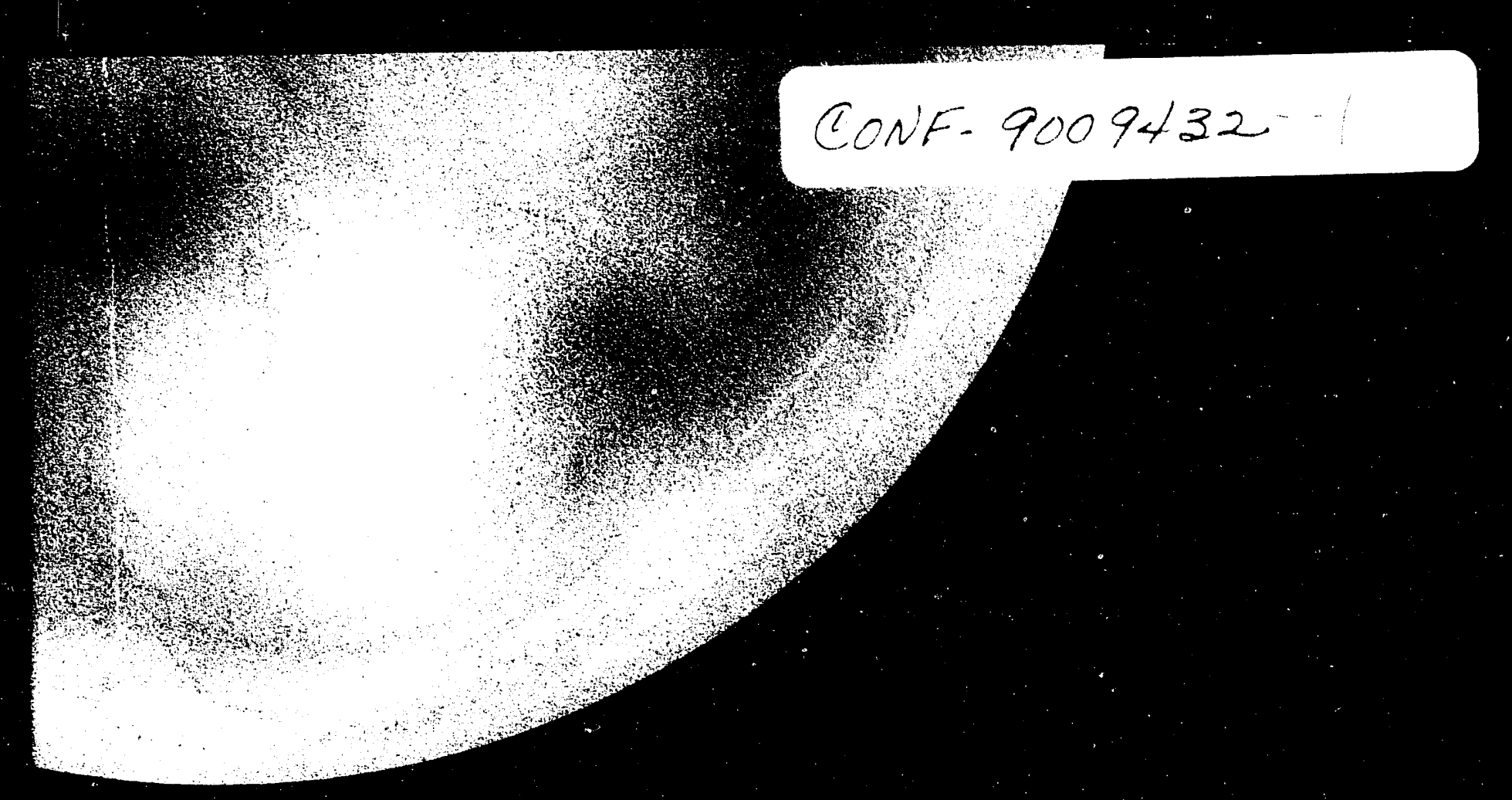

Institute of

Geophysics and

Planetary

Physics

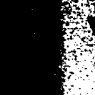

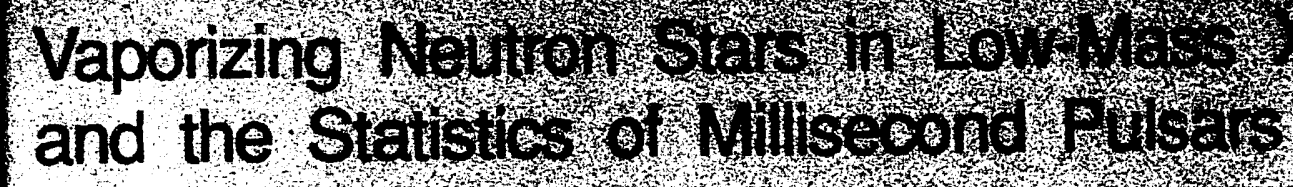

\section{Marco Tavani}

Manuscript date: August 8,1991

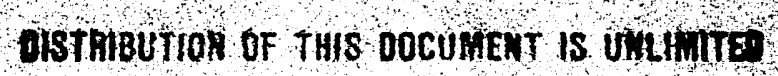

UCRL-JC- 108045

Lawrence Livermore National Laboratory

University of California 


\section{DISCLAIMER}

This document was prepared as an account of work sponsored by an agency of the United States Government. Neither the United States Government not the University of California nor any of their employees, makes any warranty, express or implied, or assumes any legal liability or responsibility for the accuracy, completeness, or usefulness of any information, apparatus, product, or process disclosed, or represents that its use would not infringe privately own rights. Reference herein to any specific commercial products, process, or service by trade name, trademark, manufacturer, or otherwise, does not necessarily constitute or imply its endorsement, recommendation, or favoring by the United States Government or the University of California. The views and opinions of authors expressed herein do not necessarily state or reflect those of the United States Government or the University of California, and shall not be used for advertising or product endorsement purposes.

This is a preprint of a paper intended for publication in a journal or proceedings.Since changes may be made before publication, this preprint is made available with the understanding that it will not be cited or reproduced without the permission of the author. 
UCRL-JC- -108045

DE92 001894

\title{
VAPORIZING NEUTRON STARS IN LOW-MASS X-RAY BINARIES AND THE STATISTICS OF MILLISECOND PULSARS
}

\author{
Marco Tavani ${ }^{1}$
}

University of California

Institute of Geophysics and Planetary Physics, LLNL, CA 94550

and

Department of Astronomy, UC Berkeley, CA 94720

Talk given at the Advanced Study Institute on

Neutron Stars: An Interdisciplinary Field

Agia Pelagia (Crete), 3-14 September 1990

to be published in the Symposium Proceedings

J. Ventura ed., Kluwer Academic Publishers

\footnotetext{
${ }^{1}$ Affiliated to the International Center of Relativistic Astrophysics (I.C.R.A.)
} 


\title{
VAPORIZING NEUTRUN STARS IN LOW-MASS X-RAY BINARIES AND THE STATISTICS OF MILLISECOND PULSARS
}

\author{
MARCO TAVANI ${ }^{1}$ \\ University of California \\ Institute of Geophysics and Planetary Physics, LLNL, CA 94550 \\ and Department of Astronomy, UC Berkeley, CA 94720.
}

$\varphi v ́ \sigma \iota \varsigma \rho \dot{\pi} \pi \tau \sigma \sigma \theta \alpha \iota \varphi \iota \lambda \varepsilon \varepsilon \tilde{\imath}$

Heraclitus

\begin{abstract}
Recent data on low-mass X-ray binaries (LMXBs) and msec pulsars (MSPs) pose a challenge to evolutionary theories which neglect the effects of disk and companion irradiation. Here we discuss the main features of a radiation-driven $(\mathrm{RD})$ evolutionary model that may be applicable to several LMXBs. According to this model, radiation from the accreting compact star in LMXBs 'vaporizes' the accretion disk and the companion star by driving a self-sustained mass loss until a sudden accretion-turn off occurs. The main characteristics of the RD-evolution are: (1) the lifetime of RD-LMXB's is of order $10^{7}$ years or less; (2) both the orbital period gap and the X-ray luminosity may be consequences of RD-evolution of LMXB's containing lower main sequence and degenerate companion stars; (3) the companion star may transfer mass to the primary even if it underfills its Roche lobe; (4) the recycled msec pulsar can continue to vaporize the low-mass companion star even after the accretion turn-off produced by a strong pulsar wind; (5) the RD-evolutionary model resolves the apparent statistical discrepancy between the number of MSP's and their LMXB progenitors.
\end{abstract}

\section{Introduction}

Heraclitus reminds us that Nature loves to be cryptic, and this is particularly true in the case of bright galactic $\mathrm{X}$-ray sources. Although there are approximately 100 known low-mass $\mathrm{X}$-ray binaries (LMXB's) in the Galaxy (and $\sim 10$ in globular clusters), in many cases the nature of the primary and companion stars, the mass transfer mechanism, and the lifetimes remain elusive. Evolutionary scenarios neglecting the effect of radiation from the primary star have been guiding the study of LMXB's, and have successfully explained the properties of several LMXB's studied individually (e.g., [1]). Depending on the nature of the Roche lobe-filling companion star, mass transfer may be driven by angular momentum loss caused by gravitational radiation (GR), magnetic braking (MB), or core expansion for sub-giant companions. In the 'standard model' the mechanism of mass transfer is independent of the nature of the primary star, and works in the same way for binaries containing black holes, neutron stars and white dwarves (cataclysmic variables, CVs). However, several questions arise both from the statistical features of LMXB's, and from new data on orbital

\footnotetext{
${ }^{1}$ Associated to the International Center of Relativistic Astrophysics (ICRA)
} 
timescales: (1) Why are the LMXB luminosities close to the Eddington limit of one solar mass primary? Why have there been no observed steady $L M X B$ 's with persistent emission in the range $L_{X} \sim 10^{36}-10^{34.5}$ erg $\mathrm{s}^{-1}$ ? In what follows we will refer to this luminosity range as the 'luminosity gap'. (2) Why is the orbital period distribution of LMXB's different from the corresponding distribution of $C V$ 's? Fig. 1 gives the orbital period distributions for LMXB's and cataclysmic variables (CV's). (3) Is the birthrate of millisecond pulsars in the Galaxy and globular clusters the same as the birthrate of their LMXB progenitors?

Here we address these questions by showing that radiation from the primary star (operating effectively only for a primary with mass-energy conversion efficiency of order $10 \%$ ) may influence binary evolution so as to produce the observed luminosity and orbital period 'gaps' of the LMXB distributions, as well as to reconcile LMXB/MSP birthrates. Some recent observations of $\dot{P}_{\text {orb }} / P_{\text {orb }}$ for the sources Cyg X-3, X 1822-371, EXO 0748-676 and $4 \mathrm{U} 1820-30$ are interpreted in terms of the RD-evolution model.

\section{Radiation-Driven Evolution of LMXB's}

The reader is referred to Refs. $[2,3,4]$ for further details of the wind formation mechanism. The RD-mass loss rate can be written as $\left|\dot{m}_{\text {rad }}\right|=10^{-17} f \hat{L} \mathrm{~g} \mathrm{~s}^{-1}$, where the dimensionless quantity $f$ depends on the details of energy deposition and radiation transport at the base of the corona, and $\hat{L}=\chi \Delta \Omega L$, where $\Delta \Omega$ is the effective solid angle subtended by the companion star to its primary, $L$ the compact star's luminosity (in erg $\mathrm{s}^{-1}$ ) and $\chi$ an attenuation factor which takes into account possible absorption/screening and scattering/reprocessing effects in the disk or corona surrounding the compact star (typically $\chi \lesssim 0.1$ for X-rays). Under certain conditions, it is possible for the mass loss to be selfsustained. In the simplest model of self-sustained evolution described here, the radiationdriven mass loss (correspoiding to a luminosity $L^{*} \propto \beta|\dot{m}|_{\text {rad }}$ ) can be either of order of $10^{18}-10^{19} \mathrm{~g} \mathrm{~s}^{-1}$ or zero. The transition occurs at a value of the companion's mass $m_{c}$ (hereafter, the critical mass) which corresponds to a sudden decrease of the accretion rate or even a permanent accretion turn-ofi if a msec pulsar has been produced [2].

\subsection{LMXB Lifetimes}

Two features of the self-sustained evolutionary phase make the lifetime of a RD-LMXB substantially shorter than in standard models: the mass loss is driven at a relatively large rate, and one which is weakly dependent on orbital evolution; and the companion may contract inside its Roche lobe and still transfer mass. This last possibility depends crucially upon the quantity $\beta$, the amount of mass lost from the binary during the RD-evolutionary phase.

The LMXB lifetime is comprised of two phases, i.e., where the companion fills and underfills its Roche lobe. We have $\tau=\Delta t_{R L}+\Delta t_{N R L}$, where the time the Roche lobe is filled is $\Delta t_{F L}=\left(m_{1} /\left\langle\left|\dot{m}_{\text {rad }}\right|\right\rangle_{R L}\right)\left(1-m^{*} / m_{1}\right)$; and the time the Roche lobe is underfilled is $\Delta i_{N R L}=\left(m^{*} /\left\langle\dot{m}_{r a d}\right\rangle_{N R L}\right)\left(1-m_{2} / m^{*}\right)$, with $m_{1}$ and $m_{2}$ respectively the initial and final companion's masses for $m_{2}<m^{*} \lesssim m_{1}$, and $\left\langle|\dot{m}|_{\text {rad }}\right\rangle$ the corresponding average mass loss rate [3]. Although the value of the $\mathrm{RD}$-mass loss rate may depend upon the orbital evolution, here we will adopt the following numerical estimates: $\left\langle\left|\dot{m}_{\text {rad }}\right|_{i R L}=\left\langle\left|\dot{m}_{\text {rad }}\right|\right\rangle_{N R L} \simeq 10^{-8} \mathrm{M}_{\odot} \mathrm{yr}^{-1} \equiv \dot{\mathrm{m}}_{8}\right.$. For LMXB's with degenerate companions, $m_{1}$ can be larger (for an initial $\beta \sim 1 / 3$ ) or even equal to $m^{*}$ (for $\beta \simeq 1$ ). Where mass transfer is self-susiained and the companion always fills its Roche lobe, $m_{2}=m_{c, R L}$, the 
critical mass being given by $m_{c, R L} \simeq(0.1 M / \Sigma) /(1-0.1 / \Sigma)$, with $\Sigma=\left(\xi_{-1} f_{-1} \beta \chi\right)^{3 / 2}$, where $\xi_{-1}=\xi / 0.1$ ( $\xi$ is the conversion of accreted matter in to energy), and $f_{-1}=f /(0.1 \Upsilon)$ with $\Upsilon$ is a dimensionless quantity depending on the irradiating spectrum (typically $\Upsilon \sim 1 / 2$ for soft gamma-rays and $\Upsilon \sim 10-10 C$ for X-rays). Alternately, after a Roche lobe underfilling evolutionary phase, $m_{2}=m_{c, N R L}=0.6 m^{*}\left[\xi_{-1} f \chi\left(m^{*} / 0.04 M_{\odot}\right)^{2 / 3}\right]^{-3 / 8}\left[1+m^{*} / M\right]^{3 / 8}$, where we used $f \sim \Upsilon$ and $m^{*} \sim 0.04 M_{\odot}$ appropriate to the evolution of binary progenitors of PSR 1957+20 [2]. Applying the relevant formulae given above to LMXB's with degenerate companions, we find the lifetime of a radiation-driven LMXB containing a companion with, say, $m_{1} \sim 0.1 M_{\odot}$ is $\tau_{d} \lesssim 10^{7}$ years for $\Upsilon \chi \sim 1$. In the case of LMXB's with degenerate companions underfilling their Roche lobe (as in the case of the progenitor of PSR $1957+20), \Delta t_{N R L} \sim 10^{6}$ years; for example, Cyg X-3 may be in this intermediate phase [5]. For the numerical estimate of the birthrate of very rapid LMXB's containing white dwarfs we will use the lifetime $\tau_{v r, 7}=\dot{m}_{8}^{-1} \tau_{v r} /\left(10^{7} \mathrm{yrs}\right)$.

Analogously, the inferred lifetime of an LMXB containing a lower main sequence companion with $n=1 / 3$ is $\sim 10^{7}$ yrs [3]. For $\beta=1$ we obtain $m_{c} / m^{*} \simeq 0.33\left(\xi_{-1} f \chi\right)^{-3 / 8} \cdot[(1+$ $\left.\left.m^{*} / M\right) /\left(m^{*} / 0.4 M_{\odot}\right)\right]^{1 / 4}$, and for $\beta=2 / 3$ we have $m_{c} / m^{*} \simeq 0.11\left(\xi_{-1} f \chi\right)^{-3 / 4}$.

$\left[\left(1+m^{*} / M\right)\left(m^{*} / 0.4 M_{\odot}\right)\right]^{1 / 2}$. In what follows, for the lifetime of rapid LMXB's we will use $\tau_{r}^{\prime}=\dot{m}_{8}^{-1} \tau_{r} /\left(3 \cdot 10^{7} \mathrm{yrs}\right)$.

\subsection{ORBITAL EVOLUTION}

The orbital evolution of an LMXB is determined by angular momentum loss (driven by gravitational radiation or a wind), as well as by mass loss from the companion star. For masses and orbital parameters of typical observed LMXB's, mass transfer mechanisms neglecting irradiation effects yield an effective lifetime of order $10^{8}-10^{9}$. Evolution driven by the combined effects of GR and MB accounts sucressfully for most of the observed properties of known CV's.

A self-sustained RD-mass loss from the companion (or from the outer edge of the disk) leads to an orbital evolution quite different from the standard model. The irradiated companion can transfer mass even if it does not fill its Roche lobe exactly. We obtain [3]

$$
\frac{\dot{P}_{\text {orb }}}{P_{\text {orb }}}=-3\left[1-(1-\beta) \tilde{\alpha}(1+q)-\beta q-\frac{1}{3}(1-\beta) \frac{q}{1+q}\right] \frac{\dot{m}_{\text {rad }}}{m}+3\left(\frac{\dot{j}}{J}\right)
$$

where $m$ is the mass of the companion star, $\dot{m}_{\text {rad }}$ the RD-mass transfer rate, $\beta$ the fraction of $|\dot{m}|$ which is accreted onto the compact star, $\dot{J} / J=(\dot{J} / J)_{G R}+(\dot{J} / J)_{M B}$ the fractional change of the orbital angular momentum $J$ from gravitational radiation (GR) and magnetic braking (MB), $q=m / M$ the ratio of the companion mass to primary mass $M$, and $\tilde{\alpha}$ the specific angular momentum parameter defined by $\delta J=\tilde{\alpha} \delta m(1-\beta) a^{2} 2 \pi / P_{\text {orb }}$, where $P_{\text {orb }}$ is the orbital period. The dimensionless parameter $\tilde{\alpha}$ gives the effective angular momentum loss caused mass loss from the binary. For example, if mass is lost from the binary at the orbital distance $a$ and if the the outflowing gas does not acquire any additional specific angular momentum, then $\bar{\alpha}=1$. For a self-sustained value of the mass loss rate the first term is the dominant one. Note that the quantity $\dot{P}_{\text {orb }} / P_{\text {orb }}$ is expected to be $\sim+10^{-6} \mathrm{yr}^{-1}$ for RD-LMY Bs with very low mass degenerate companions. For main sequence companions the time derivative of the orbital period may be either positive or negative depering primarily on the value of $\beta$ and $\tilde{\alpha}$. The associated lifetime turns out to be $\sim 10^{7}$ years. 


\subsection{ORBITAL PERIOD 'GAP' OF LMXB's}

The LMXB orbital period gap can be 'crossed' relatively rapidly by RD-systems containing degenerate companions, or never crossed and 'repelled' by RD-binaries with main sequence companions. Alternately, even if the mechanism of mass transfer is not driven by radiation during the evolution of LMXB's above the period gap (for $P_{\text {orb }} \gtrsim 3$ hours), the sudden quenching of mass trasfer occurring at $P_{\text {orb }} \sim 3$ hours may yield a millisecond pulsar whose radiation pressure could be large enough to stop any further accretion. Fig. 1 shows the schematic orbital behavior expected in the RD-evolution.

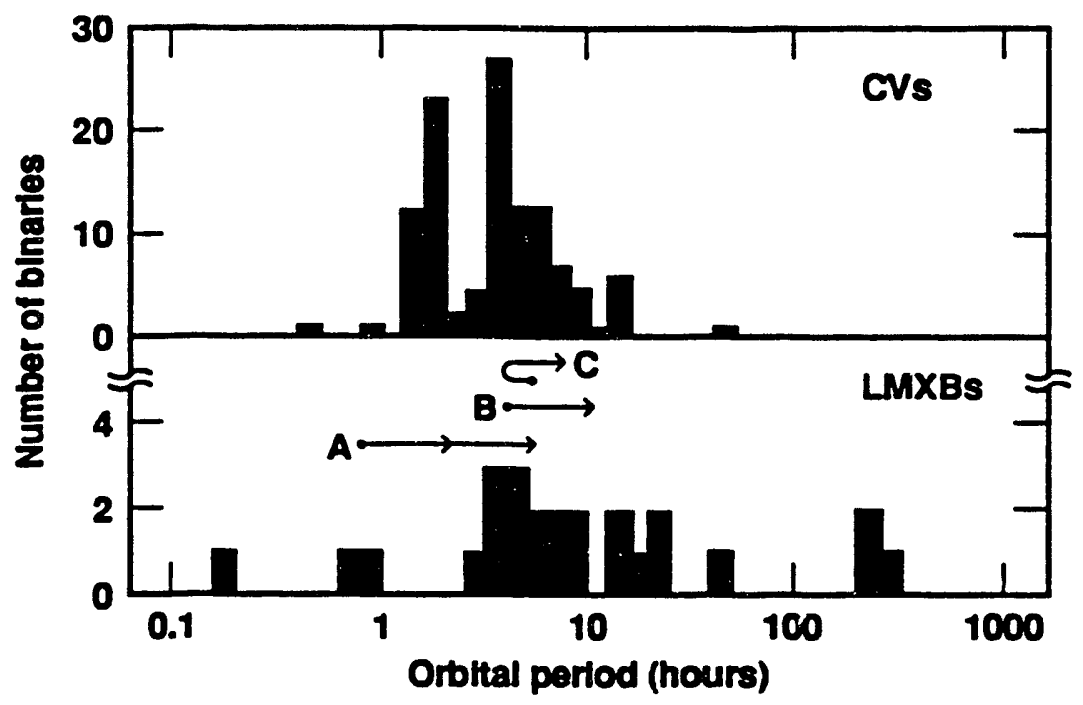

FIGURE 1 Distribution of orbital periods of CVs and LMXBs. Solid curves give the schematic behavior of the orbital RD-evolution. Line $A$ represents the rapid expansion of an LMXB with a degenerate companion for $\beta$ in the range $1 / 3 \lesssim \beta \lesssim 1$; the timescale may be $\sim 10^{6}$ yrs. Line $B$ and line $C$ give the orbital evolution according to $\mathrm{Eq}$. (1) of LMXBs with main sequence companions.

\subsection{VAPORIZING MILLISECOND PULSARS}

Even in the post-accretion phase, radiation produced by the relativistic wind emanating from millisecond pulsars can continue to vaporize and drive mass loss from their low-mass companions $[2,3,4]$ leading to partial or even total evaporation of the companion star. The ratio of the evaporation time scale $\tau_{\text {evap. }}=m_{c} /\left|\dot{m}_{\text {rad }}\right|$ and the spin-down time scale defined as $\tau_{s}=P / \dot{P}$ with $P$ the pulsar spin period and $\dot{P}$ its time derivative is

$$
\frac{\tau_{\text {evap }}}{\tau_{s}} \simeq 0.8 \frac{P_{-3}^{2}}{(f / 0.1) I_{45}}\left(\frac{m_{c}}{0.2 M_{\odot}}\right)^{1 / 3} \frac{1}{\Psi_{c}^{2}}
$$

where $P_{-3}$ is the spin period in milliseconds, $I_{45}$ the neutron star's moment of inertia in units of $10^{45} \mathrm{~g} \mathrm{~cm}^{2}, \Psi_{c}$ the value of $\Psi=R / R_{L}$ at the accretion turn-off with $R_{L}$ the Roche lobe radius. In Eq. (2) the total mass has been assumed to be $\sim 1.8 M_{\odot}$. Energy deposition in the irradiated companion's atmosphere by secondary radiation produced by the pulsar relativistic wind ${ }^{2}$ gives [2] $f \simeq 0.01 \Upsilon$. Even though complete evaporation of

\footnotetext{
${ }^{2}$ In the case of PSR $1957+20$, whether the ratio $\tau_{\text {evap. }} / \tau$, is less than unity depends upon currently unknown details of the irradiating spectrum; we obtain $\tau_{\text {evap }} / \tau_{0} \simeq 0.8 f^{-1} I_{13}^{-1}$. For a synchtrotron spectrum at fixed electron energy, $\Upsilon \simeq 10$, and for a time in tegrated synchrotron spectrum of $e^{ \pm}$pairs which lose most of their energy, $\Upsilon \simeq 100$ [2]. It is therefore plausible that the companion of $1957+20$ would be completely
} 
low-mass companions is a possible outcome of RD-evolution, only a subclass of MSP's are capable of producing a sufficiently large evaporative wind. The RD-evolution of LMXB's makes plausible the existence of a relatively large number of MSP's in detached binaries containing lower main sequence stars heated and probably slightly evaporated by the pulsar wind. Doppler-induced spread caused by orbital motion in binaries with $P_{\text {orb }}$ of order of a few hours makes difficult the detection of this important class of binary millisecond pulsars. However, the detection of MSP's with main sequence companions would provide invaluable information about binary evolution. It could be possible to distinguish between models which neglect companion irradiation and predict binary MSP's with $P_{\text {orb }} \simeq 3 \mathrm{hrs}$, and the RD-model, which predicts $3 \mathrm{hr} \lesssim P_{\text {orb }} \lesssim 10 \mathrm{hr}$.

\section{Statistics of Millisecond Pulsars and LMXB's}

A recent statistical analysis of galactic MSP's gives a birthrate $B R(M S P s) \simeq 2 \cdot 10^{-5} y r^{-1}$ after taking into account the selection effects associated with radiopulsar surveys [7]. This birthrate is about 1000 times smaller than the galactic supernova formation rate and is too large by a factor $\sim 10-100$ to be in agreement with standard evolutionary models of LMXB's. This discrepancy is even more pronounced for the subgroup of '. . jid' lowmass binary pulsar systems (LMBP's), where the orbital period is $P_{\text {orb }} \lesssim 10$ days, and the birthrate $B R($ rapid $L M B P s) \simeq 1.5 \cdot 10^{-5} \mathrm{yr}^{-1}$. It is appropriate to consider separately binaries defined as 'very rapid' ( $P_{i} \lesssim 0.5$ days), 'rapid' $\left(0.5\right.$ days $\lesssim P_{\mathrm{i}} \lesssim 3$ days), and 'slow' ( $P_{i} \gtrsim 3$ days), with $P_{i}$ the initial orbital period. From the estimates of section 2.1 we can calculate the birthrates of rapid LMXB's evolving due to the radiation-driven mechanism of mass transfer. We obtain [9] $B R\left(\right.$ very rapid $\left.L M X B^{\prime} s\right) \simeq\left(7 \cdot 10^{-6} y r^{-1}\right) f_{w} \tau_{v r, 7}^{-1}$ and $B R\left(\right.$ rapid $\left.L M X B^{\prime} s\right) \simeq\left(6 \cdot 10^{-7} y r^{-1}\right) f_{r} \tau_{r}^{\prime-1}$, with $f_{w} \simeq 1 / 2$ the fraction of very rapid LMXB's with white dwarf and main sequence companions, and $f_{r}$ the fraction of LMXB's with $P_{\text {orb }} \gtrsim 0.5$ days containing sub-giant companions as defined in Ref. [7]. If we compare the birthrate of very rapid LMXB's driven by radiation and of rapid MSP's we find that they are approximately equal. Given the current statistical uncertainties of pulsar surveys and LMXB number estimations the statistical MSP/LMXB discrepancy may therefore be resolved by very rapid RD-LMXB's [9]. We note that since the 'prompt' formation of MSP's after the AIC of white dwarf primaries possibly affects only a fraction of slow binaries, the AIC mechanism is insignificant in this context.

At present it is uncertain whether the MSP/LMXB discrepancy in globular clusters is the same as in the Galaxy [8] or smaller by a factor of $\sim 10$ [10]. The inferred MSP birthrate is $B R($ cluster $M S P s) \simeq(0.2-1.7) \cdot 10^{-6} \mathrm{yr}^{-1}$. From the measured value of $\dot{P}_{\text {orb }} / P_{\text {orb }}$ for $4 \mathrm{U}$ 1820-30 (see Table 1) and the large value of the mass loss rate of AC211 in the globular cluster $\mathrm{M} 15$, we obtain $B R(H M L-L M X B s) \simeq 1 \cdot 10^{-6} \mathrm{yr}^{-1}$, if we consider only the two high-mass-loss (HML) binaries $4 U$ 1820-30 and AC211. We note that despite their apparent low X-ray luminosities, LMXB's in globular clusters may have RD-driven orbital timescales of order $10^{7} \mathrm{yrs}$, similarly to the X-ray burster EXO 0748-676 (see Table 1). The RD-LMXB birthrate in globular clusters may well be $\gtrsim$ a few $10^{-6} \mathrm{yr}^{-1}$.

evaporated even if $\Psi_{c} \simeq 1 / 3$. However, for the eclipsing pulsar in Ter 5 PSR 1744-24A we have $\tau_{\text {evap. }} / \tau_{s} \gg 1$. We note that the discovery probability of binary pulsars not able to completely vaporize their companion is much larger than the probability of discovering systems with $\tau_{\text {evap. }} / \tau_{\text {s }} \ll 1$. 
Table 1

\begin{tabular}{||l|c|l|c|c||}
\hline \hline LMXB & $P_{\text {orb }}$ (hours) & $\dot{P}_{\text {orb }} / F_{\text {orb }}\left(\mathrm{yr}^{-1}\right)$ & timescale (years) & Ref. \\
\hline Cyg X-3 & 4.82 & $+(2.20 \pm 0.22) \cdot 10^{-6}$ & $5 \cdot 10^{5}$ & 11 \\
X 1822-371 & 5.57 & $+(3.40 \pm 0.94) \cdot 10^{-7}$ & $2.9 \cdot 10^{6}$ & 12 \\
EXO 0748-676 & 3.82 & $-(2.02 \pm 0.28) \cdot 10^{-7}$ & $5 \cdot 10^{6}$ & 13 \\
4U 1820-30 & 0.18 & $-(1.08 \pm 0.19) \cdot 10^{-7}$ & $10^{7}$ & 14 \\
\hline \hline
\end{tabular}

\section{Observation vs. Theory}

The quantity $\dot{P}_{\text {orb }}$ has now been measured for Cyg X-3 [11], X 1822-371 [12], EXO 0748-676 [13], and $4 \mathrm{U} 1820-30$ [14], all of which have $P_{\text {orb }} \lesssim 5.6$ hours. Table 1 gives the properties of the four LMXB's whose $\dot{P}_{\text {orb }} / P_{\text {orb }}$ has been measured. None of the measured values agrees with the predictions of the standard GR and MB models. The inferred timescale for orbital evolution is about 100 times shorter than expected in all systems. However, the RDevolutionary mechanism may explain the observations quite naturally [6]. Cyg X-3 fits the evolutionary scenario for a RD-LMXB containing a very low mass white dwarf companion $[5,3]$. RD-LMXB's with lower main sequence companions may be characterized by a large positive value of $\dot{P}_{\text {orb }} / P_{\text {orb }}$ if the mass loss from the companion is $\dot{m}_{8} \gtrsim 3$; and this may be the case for $X$ 1822-371. Alternately, X 1822-371 may contain a white dwarf companion, and in this case the likely companion mass is $m \simeq\left(0.1 M_{\odot}\right) \dot{m}_{8}$. We note that both the sign and magnitude of $\dot{P}_{\text {orb }} / P_{\text {orb }}$ for Cyg X-3 and X 1822-371 suggest a stable RD-mass transfer with $1 / 3 \lesssim \beta \lesssim 1$ and $\Psi \lesssim 1$. By contrast, EXO 0748-676 and $4 \mathrm{U} 1820-30$ may belong to a class of RD-LMXB's in which the mass loss from the binary plays a crucial role in determining the orbital evolution [6]. According to the RD-evolution model, EXO 0748676 and $4 \mathrm{U} 1820-30$ are characterized by a relatively large mass loss from the binary with $\beta \sim 1 / 10$ and $\Psi \gtrsim 1$.

Work performed at IGPP-LLNL under the auspices of the U.S. DOE under contract W7405-ENG-48.

\section{References}

[1] Rappaport, S., Verbunt, F., and Joss, P.C., 1983, Ap.J, 275, 713.

[2] Tavani, M., 1989, in Proc. of the 23rd ESLAB Symposium, ESA SP-296, Vol. 1, p. 241.

[3] Tavani, M., 1990, submitted to Ap.J.

[4] Shaham, J., 1990, these Proceedings.

[5] Tavani, M., Ruderman, M. and Shaham, 1989, Ap. J.(Letters), 342, L31.

[6] Tavani, M., 1990, submitted to Nature.

[7] Kulkarni S.R., and Narayan, R., 1988, Ap.J, 335, 755.

[8] Kulkarni, S., Narayan, R., and Romani, R.W., 1990, Ap.J., 356, 174.

[9] Tavani, M., 1991, Ap.J. (Letters), 366, L27-L31.

[10] Fruchter, A., Goss, D., 1990, Ap.J. (Letters), 365, L63-L66.

[11] Kitamoto, S., Miyamoto, S., and Matsui, W., 1987, P.A.S.J., 39, 259.

[12] Hellier, C., Mason, K.O., Smale, A.P., Kilkenny, D., 1990, M.N.R.A.S., 244, 39P.

[13] Parmar, A.N., Verbunt, F., Smale, A.P., and Corbet, R.H.D., 1991, Ap.J., 366, 253.

[14] Tan, J.. et al., 1990. submitted to Ap. J. 

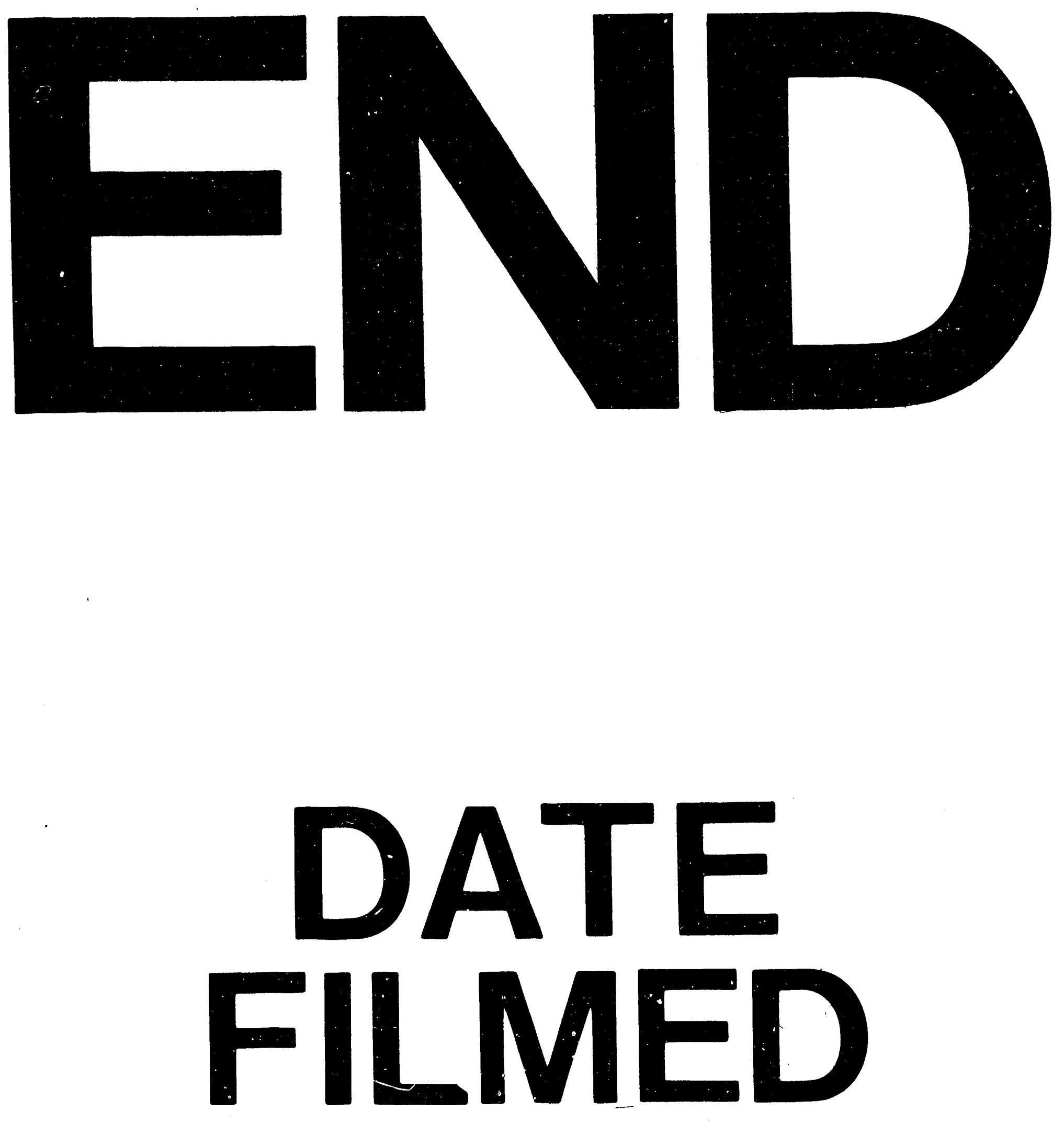

I

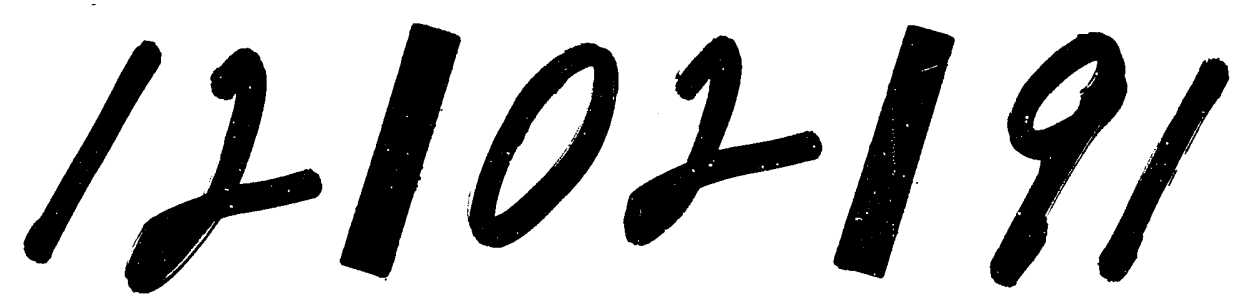




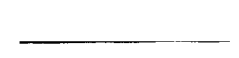

